\title{
Millimeter-wave compressive holography
}

\author{
Christy Fernandez Cull, ${ }^{1, *}$ David A. Wikner, ${ }^{2}$ Joseph N. Mait, ${ }^{2}$ \\ Michael Mattheiss, ${ }^{3}$ and David J. Brady ${ }^{1}$ \\ ${ }^{1}$ Fitzpatrick Institute for Photonics and Department of Electrical and Computer Engineering, \\ Duke University, 129 Hudson Hall, Durham, North Carolina 27708, USA \\ ${ }^{2}$ United States Army Research Laboratory, 2800 Powder Mill Road, \\ Adelphi, Maryland 20783, USA \\ ${ }^{3}$ Department of Electrical and Computer Engineering, University of Maryland, \\ 2405 A.V. Williams Building, College Park, Maryland 20742, USA \\ *Corresponding author: caf11@ee.duke.edu
}

Received 16 December 2009; revised 26 March 2010; accepted 16 April 2010; posted 19 April 2010 (Doc. ID 121568); published 12 May 2010

\begin{abstract}
We describe an active millimeter-wave holographic imaging system that uses compressive measurements for three-dimensional (3D) tomographic object estimation. Our system records a two-dimensional (2D) digitized Gabor hologram by translating a single pixel incoherent receiver. Two approaches for compressive measurement are undertaken: nonlinear inversion of a 2D Gabor hologram for 3D object estimation and nonlinear inversion of a randomly subsampled Gabor hologram for 3D object estimation. The object estimation algorithm minimizes a convex quadratic problem using total variation (TV) regularization for $3 \mathrm{D}$ object estimation. We compare object reconstructions using linear backpropagation and TV minimization, and we present simulated and experimental reconstructions from both compressive measurement strategies. In contrast with backpropagation, which estimates the 3D electromagnetic field, TV minimization estimates the $3 \mathrm{D}$ object that produces the field. Despite undersampling, range resolution is consistent with the extent of the 3D object band volume. () 2010 Optical Society of America

OCIS codes: $\quad 090.1995,100.3200,100.6950,110.1758,110.3010,110.3200$.
\end{abstract}

\section{Introduction}

Various methods exist for concealed weapons detection [1]. These methods aim to penetrate common obstructions such as clothing or plastics. X-ray [2] and millimeter-wave (MMW) [3] imaging systems are technologies capable of penetrating these barriers for imaging suicide bomb vests or weapons composed of metals, nonmetals, or plastics. While x-ray imaging capabilities are highly effective, questions about health risks impair the feasibility of such systems for real-time imaging. MMW for low-power (on the order of $\mathrm{mW}$ ) imaging systems do not present a health hazard and therefore enable real-time imaging of targets with high contrast and high resolution.

0003-6935/10/190E $67-16 \$ 15.00 / 0$

(C) 2010 Optical Society of America
Several studies have explored both active and passive MMW imagers for concealed weapons detection $[4,5]$ where the system limitation is the detector array cost. Some systems include portal, or handheld devices [6] operating in close range to the target. Other systems are holographic [7,8]. These MMW focal and interferometric systems map object information onto a two-dimensional (2D) or a linear array that is typically scanned for image formation. These scanning systems [9-11] are plagued by their associated data acquisition times. For these systems, there is a trade-off between scan time and measurement signal-to-noise ratio (SNR). Therefore, rapid scanning of concealed weapons is challenging for current MMW systems.

For stand-off explosive detection, rapid scanning of the target is a necessity. To overcome the bottleneck associated with current MMW scanning systems, we consider compressive sensing (CS). Recent studies in 
CS reveal that an $N$-point image can be restored from $M$ measurements, where $M \ll N$ [5, 12-14]. Chan et al. [13] used a focal system to randomly sample spatial frequencies in the Fourier plane for 2D object estimation at $100 \mathrm{GHz}$. We are further motivated to investigate CS for MMW imaging based on similar work in $633 \mathrm{~nm}$ compressive holography [15]. Holography, which measures a limited set of spatial frequencies in the Fourier domain, is a compressive encoder, because it compresses three-dimensional (3D) spatial information into a single interferometric planar field. Since the entire extent of an object's 3D spatial frequency band volume can not be captured in a single exposure, multiangle illumination or object rotation is typically used to improve $3 \mathrm{D}$ object estimation. The results in [15] suggest that $3 \mathrm{D}$ tomographic estimation can be achieved from a $2 \mathrm{D}$ hologram recording.

This paper extends compressive holography to millimeter wavelengths. The subject matter in this paper differs from compressive holography at visible wavelengths, because sparse holographic sampling is implemented to minimize the data acquisition scan cost associated with imaging at millimeter wavelengths. Also, the MMW holography system operates in a completely different wavelength region with corresponding differences in optics and detectors, so it required a completely new system design compared to work at visible wavelengths. Further, in this paper, we holographically sample a subset of spatial frequencies for 3D object estimation. Also, we randomly subsample a $2 \mathrm{D}$ hologram to further analyze the impact of fewer measurements on 3D object estimation. Our holographic technique is similar to [8], as we are not band limited by a lens aperture and phase information is preserved. We differ in our nonlinear inversion approach for 3D object estimation. Our method optimizes a convex quadratic problem using total variation (TV) regularization. Unlike classical reconstruction with backpropagation, we demonstrate that undiffracted fields, overlaid in the frequency domain of a Gabor hologram, can be separated by imposing a TV sparsity constraint.

Although other contributions in the literature embody a mathematical framework similar to compressive holography [16-18], there exists a fundamental difference in philosophy. Compressive holography exploits encoding and undersampling for 3D object estimation, whereas techniques in diffraction tomography are designed to overcome sampling limitations imposed by the data collection process. Also, recent work by Denis et al. [19] presents a similar twin-image suppression method; however, a sparsity-enforcing prior in a Bayesian framework combined with 11 regularization is used for object estimation. Our work represents the confluence of MMW digitized holographic measurement and TV minimization for 3D object estimation with minimal error. We adapted the algorithm framework of [15] for sparse holographic sampling and data inversion.
This paper is organized as follows. In Section 2, we describe the theoretical background for diffraction tomography and holographic measurement. Hologram recording geometry and resolution metrics are also discussed in this section. Section 3 summarizes our TV minimization algorithm used for $3 \mathrm{D}$ object estimation from a 2D digitized composite hologram. Also, we present simulated data of randomly subsampled $2 \mathrm{D}$ holograms to analyze the impact of fewer measurements on 3D object estimation. Section 4 describes the experimental platform. Section 5 presents TV minimization and backpropagation reconstructions. Finally, in Section 6, we provide a summary of the results and concluding remarks.

\section{Theory}

Our ultimate goal is to make the smallest number of measurements about a $3 \mathrm{D}\left(x^{\prime}, y^{\prime}, z^{\prime}\right)$ object $f_{o}\left(\mathbf{r}^{\prime}\right)$, where $\mathbf{r}^{\prime}$ is a $3 \mathrm{D}$ spatial vector, such that it is possible to reconstruct $f_{o}\left(\mathbf{r}^{\prime}\right)$ with minimal error. Rather than attempting to form an image of $f_{o}\left(\mathbf{r}^{\prime}\right)$ point by point, our approach is based on making measurements in the far field where spatial frequencies $\left(u_{x}\right.$ and $\left.u_{y}\right)$ are measured. To do this, we record a hologram. A hologram $g\left(\mathbf{r}_{\mathbf{h}}\right)$ is a record of the interference between two wave fields, a reference field $E_{r}(\mathbf{r})$ and an object scattered field $E_{o}(\mathbf{r})$. To record a hologram, a squarelaw detector in the hologram plane $\mathbf{r}_{\mathbf{h}}=\left(x, y, z_{h}\right)$ measures a time-averaged intensity of the interference:

$$
\begin{aligned}
g\left(\mathbf{r}_{\mathbf{h}}\right)= & \left|E_{r}\left(\mathbf{r}_{\mathbf{h}}\right)+E_{o}\left(\mathbf{r}_{\mathbf{h}}\right)\right|^{2}=\left|E_{r}\left(\mathbf{r}_{\mathbf{h}}\right)\right|^{2}+\left|E_{o}\left(\mathbf{r}_{\mathbf{h}}\right)\right|^{2} \\
& +2\left|E_{r}\left(\mathbf{r}_{\mathbf{h}}\right) E_{o}\left(\mathbf{r}_{\mathbf{h}}\right)\right| \cos \left[\theta_{r}\left(\mathbf{r}_{\mathbf{h}}\right)-\theta_{o}\left(\mathbf{r}_{\mathbf{h}}\right)\right],
\end{aligned}
$$

where $\theta_{r}\left(r_{h}\right)$ represents the phase associated with the propagated reference wave field and $\theta_{o}\left(\mathbf{r}_{\mathbf{h}}\right)$ represents the phase associated with the propagated object wave field. We assume our object field is generated by illuminating a $3 \mathrm{D}$ object $f_{o}\left(\mathbf{r}^{\prime}\right)$ by an on-axis plane wave $\exp \left(-2 \pi i \mathbf{u}_{\mathbf{o}} \cdot \mathbf{r}\right)$, where $\mathbf{u}_{\mathbf{o}}=\left(u_{x_{o}}, u_{y_{o}}, u_{z_{o}}\right)$. The 3D object $f_{o}\left(\mathbf{r}^{\prime}\right)$ represents an object scattering amplitude where after reference plane wave illumination, a fraction of the energy is either transmitted or reflected at a point in $3 \mathrm{D}$ space. We do not assume the object induces any phase change in an incident wave field through polarization or birefringence.

If the object is transmissive and located at $z_{h}$ distance away from the hologram plane, under the Born approximation the scattered field is

$$
E_{o}\left(\mathbf{r}_{\mathbf{h}}\right)=\frac{-\pi}{\lambda^{2}} \int E_{r}\left(\mathbf{r}^{\prime}\right) f_{o}\left(\mathbf{r}^{\prime}\right) h\left(\mathbf{r}_{h}-\mathbf{r}^{\prime}\right) \mathrm{d} \mathbf{r}^{\prime}
$$

where $h\left(\mathbf{r}_{\mathbf{h}}-\mathbf{r}^{\prime}\right)$ is the shift-invariant impulse response and $E_{r}\left(r^{\prime}\right)$ is the reference plane wave. For scalar waves in homogeneous space, the impulse response is

$$
h\left(\mathbf{r}_{\mathbf{h}}-\mathbf{r}^{\prime}\right)=\frac{\exp \left(2 \pi i\left|\mathbf{r}_{\mathbf{h}}-\mathbf{r}^{\prime}\right| / \lambda\right)}{\left|\mathbf{r}_{\mathbf{h}}-\mathbf{r}^{\prime}\right|} .
$$


We can reformulate the convolution integral in Eq. (2) using the Fourier convolution theorem. The Fourier transform of the scattered field along the transverse axes in the recording plane is

$$
\begin{aligned}
\hat{E}_{0}\left(u_{x}, u_{y}, z_{h}\right) & =\frac{1}{i \pi \lambda} \hat{f}_{o}\left(u_{x}-u_{x_{o}}, u_{y}-u_{y_{o}}\right. \\
& \left.\times \sqrt{\frac{1}{\lambda^{2}}-u_{x}^{2}-u_{x}^{2}}-u_{z_{0}}\right) G_{2 D}\left(u_{x}, u_{y}, z\right),
\end{aligned}
$$

where

$$
G_{2 D}\left(u_{x}, u_{y}, z\right)=\frac{\exp \left(2 \pi i z \sqrt{\frac{1}{\lambda^{2}}-u_{x}^{2}-u_{y}^{2}}\right)}{\sqrt{\frac{1}{\lambda^{2}}-u_{x}^{2}-u_{y}^{2}}}
$$

$\hat{f}_{o}$ is the 3D Fourier-transform of the object density, and the exponential term represents a propagation transfer function. Under the small angle approximation, $u_{z}=1 / \lambda$ and $u_{x}, u_{y} \leq 1 / \lambda$. The frequencydomain scattered field is then approximated by

$$
\begin{aligned}
\hat{E}_{o}\left(u_{x}, u_{y}, z\right)= & \frac{1}{i \pi \lambda} \hat{f_{o}}\left(u_{x}-u_{x_{o}}, u_{y}-u_{y_{o}},-\frac{\lambda}{2}\left(u_{x}^{2}+u_{y}^{2}\right)\right) \\
& \times \exp \left(2 \pi i z \sqrt{\frac{1}{\lambda^{2}}-u_{x}^{2}-u_{y}^{2}}\right) .
\end{aligned}
$$

As discussed in Section 3, digital processing of the Gabor hologram aims to isolate the scattered field, $E_{o}\left(\mathbf{r}_{\mathbf{h}}\right)$, signal term from background and conjugate terms. If we assume the recorded hologram measures $E_{o}\left(\mathbf{r}_{\mathbf{h}}\right)$ directly and that $E_{r}\left(\mathbf{r}_{\mathbf{h}}\right)=1$, Eq. (6) demonstrates that a $2 \mathrm{D}$ hologram captures a $3 \mathrm{D}^{-}$parabolic slice of the object's band volume. Figure 1 describes tomographic sampling of a 3D band volume in a Gabor geometry. Typically, the illumination (or the object) must be rotated to fully sample the 3D band volume. To increase longitudinal resolution, the system may alternatively be scanned in frequency. Instead of scanning in the frequency domain on the surface of a sphere, this approach allows one to scan

a spherical shell with radii corresponding to a wavelength range. In [15], we showed that one may apply CS theory to estimate the $3 \mathrm{D}$ distribution of $f\left(\mathbf{r}^{\prime}\right)$ from a single holographic image without scanning in frequency or rotating the object. Instead, we exploit sparsity. This work extends 3D tomographic estimation from $2 \mathrm{D}$ holographic measurements to millimeter wavelengths.

\section{A. Hologram Recording Geometry}

The geometry used to record the hologram impacts the postdetection signal processing and the ability to reconstruct the image. In an off-axis geometry [20], the signal and its conjugate are separated from each other in frequency space and from the on-axis undiffracted energy [see Fig. 2(a)].

Note that the maximum spatial frequency $u_{\max }$ that the detector can record is limited by the sampling pitch $(d x)$ of the detector:

$$
u_{\max }=\frac{1}{2 d x} .
$$

With our detector, the pixel pitch is set by the WR-08 waveguide size $(2.32 \mathrm{~mm} \times 1.08 \mathrm{~mm})$. The maximum spatial frequency recorded in the vertical direction is $0.463 \mathrm{~mm}^{-1}$, and in the horizontal direction it is $0.216 \mathrm{~mm}^{-1}$.

Figure 2(a) shows that the information content of the object and the pixel pitch of the detector impose minimum and maximum limits on the angle $\theta_{c z}$ of the off-axis reference. For simplicity, we assume the reference beam has no $y$ component. To separate the object from its squared magnitude without ambiguity due to detector aliasing or from confusion with undiffracted terms, the angle of the off-axis beam must satisfy

$$
\theta_{c \min } \leq \theta_{c z} \leq \theta_{c \max }
$$

where

$$
\theta_{c \max }=\sin ^{-1}\left[\left(\frac{\lambda}{2}\right)\left(\frac{1}{d x}-u_{B}\right)\right]
$$

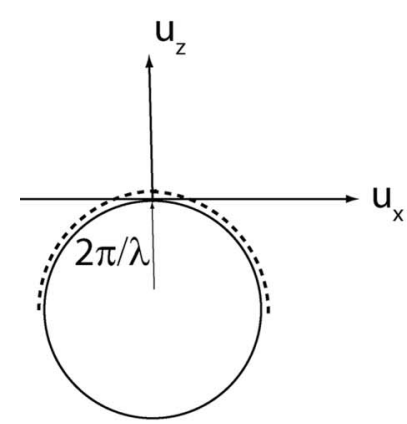

(a)

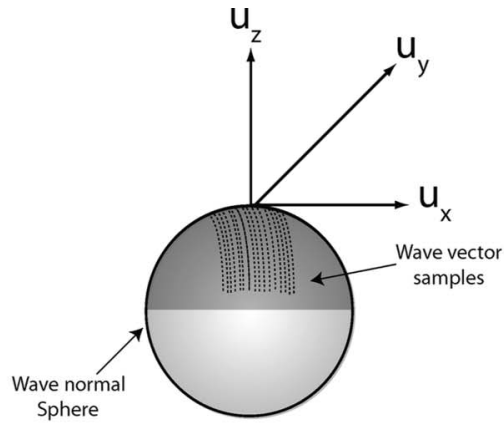

(b)

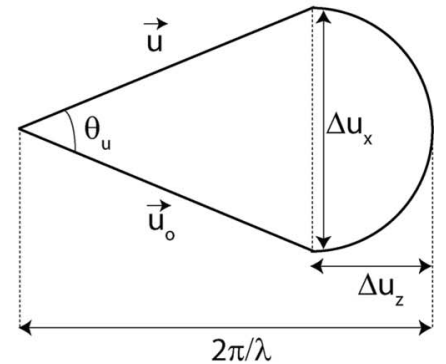

(c)

Fig. 1. Fourier-transform domain sampling of the object band volume in a transmission geometry. (a) $2 \mathrm{D}$ slice of a $3 \mathrm{D}$ sphere where the dotted curve represents the measurement from single plane wave illumination. (b) Rectilinear pattern represents wave vectors sampled by the hologram due to a finite detector plane sampling. (c) Wave normal sphere cross section for spatial and axial resolution analysis. 


$$
\theta_{c \min }=\sin ^{-1}\left(3 \frac{\lambda u_{B}}{2}\right),
$$

and $u_{B}$ is the spatial frequency bandwidth of the object.

Use of an off-axis reference beam simplifies our reconstruction because digital signal processing allows one to yield an estimate of the object field

$$
\tilde{g}_{\text {offaxis }}=E_{r}\left(\mathbf{r}_{\mathbf{h}}\right)^{*} E_{o}\left(r_{h}\right)
$$

In an on-axis geometry, it is more difficult to separate the object field from the undiffracted, zero-order fields and from its conjugate. The overlap of these three fields degrades resolution and contrast in the object reconstruction. One can apply DC suppression techniques to enhance object reconstructions [21-23], and measurements of the energy in the reference beam alone can be made and subtracted from the hologram:

$$
\tilde{g}_{\text {onaxis }}=\left|E_{o}\left(\mathbf{r}_{\mathbf{h}}\right)\right|^{2}+E_{r}^{*}\left(\mathbf{r}_{\mathbf{h}}\right) E_{o}\left(\mathbf{r}_{\mathbf{h}}\right)+E_{r}\left(\mathbf{r}_{\mathbf{h}}\right) E_{o}^{*}\left(\mathbf{r}_{\mathbf{h}}\right) .
$$

In this paper, we record a hologram in an on-axis geometry because the need for an increased bandwidth in the off-axis case outweighs the complexity for on-axis object isolation.

\section{B. Hologram Plane Sampling and Resolution Metrics}

In our implementation, the field $g\left(\mathbf{r}_{\mathbf{h}}\right)$ is sampled and digitized into a $2 \mathrm{D}$ matrix by translating a point detector in $x$ and $y$ at the hologram plane $z_{h}$. An analytical discussion of the discrete model is detailed in [15] and addressed in Section 3.

Holographic measurements captured digitally, by a scanning detector, are related to measurements made in the spatial frequency domain. The total number of detector measurements $N$ is

$$
N=n_{x} n_{y}
$$

where $n_{x}$ and $n_{y}$ represent the number of measurements along each spatial dimension in $x$ and $y$. Given the detector sampling pitch $(d x)$, the number of measurements $\left(n_{x}\right)$ in the hologram plane along the horizontal dimension is given by

$$
n_{x}=\frac{W_{x}}{d x}
$$

where

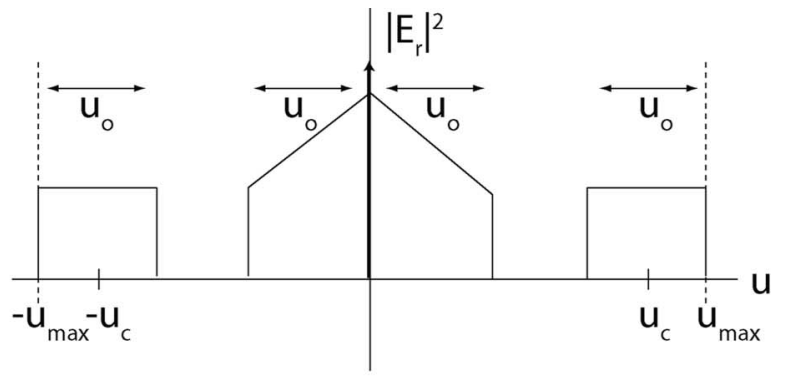

(a)



(b)

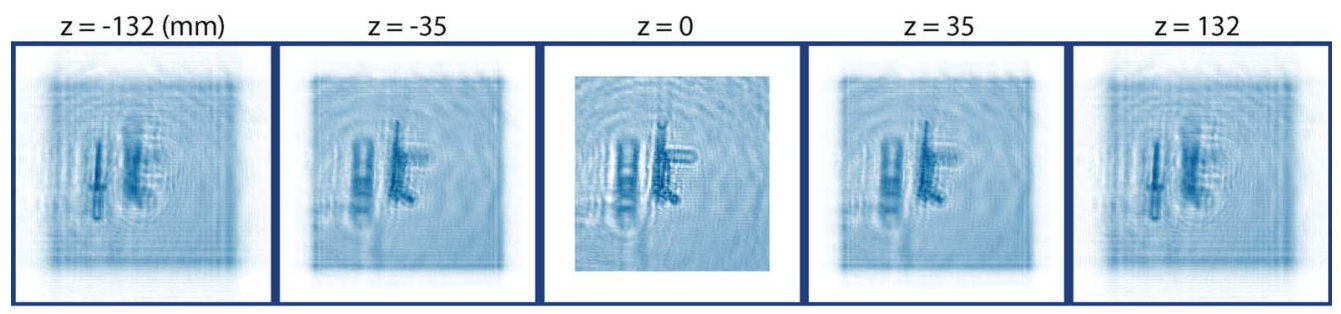

(c)

Fig. 2. (Color online) (a) Spectrum for an off-axis hologram recording depicting an inherent increase in bandwidth for adequate object separation from undiffracted terms. (b) Spectrum for a Gabor hologram recording, depicting the overlay of undiffracted, object, and conjugate terms. (c) Transverse slices from linear inverse propagation results at various $z$ planes. 


$$
W_{x}=\frac{\lambda z}{\Delta x_{o}},
$$

$z$ is the distance between the object and the detector, $\Delta x_{o}$ is the one-dimensional spatial resolution with which we wish to image the object, and $W_{x}$ refers to the spatial extent of a diffracted object $\left(\Delta x_{o}\right)$. The inverse scaling relationship arises from the conjugate relationship between the object and the hologram [24].

Detector sampling over a finite field size affects sampling resolution in the frequency domain. The sampling resolution, $\Delta_{u}$, in the frequency domain along both the horizontal and vertical dimensions $\left(u_{x}\right.$ and $u_{y}$ ), assuming $n_{x}=n_{y}$, is determined by the sampling field size at the detector plane, $\Delta_{u}=$ $1 /\left(2 n_{x} d x\right)$. The maximum spatial frequency sampled by the detector is equal to $u_{\max }$ in Eq. (7).

Resolution metrics, lateral $\left(\Delta_{x}\right)$ and axial $\left(\Delta_{z}\right)$, for the Gabor geometry are determined by the illumination wavelength and the system numerical aperture (NA). Based on the Gabor recording geometry, the NA is defined by

$$
n \sin \theta_{u}=\frac{W_{x}}{2 z}=\frac{\lambda}{2 \Delta x_{o}},
$$

where $n$ is the refractive index of air and $\theta_{u}$ is the half-angle subtended by the object to half the spatial extent of the hologram plane $\left(W_{x} / 2\right)$. Recall that the spatial resolution is related to the inverse scaling relationship in the frequency domain. The half-angle, $\theta_{u}$, is also defined as the angular bandwidth sampling on the wave normal sphere due to system NA. Thus, the NA can also be described in the spatial frequency domain. The wave vector geometry for hologram recording is shown in Figs. 1(b) and 1(c). Considering the geometry in Fig. 1(c), we write

$$
\sin \left(\theta_{u}\right)=\frac{\Delta u_{x}}{|\mathbf{u}|},
$$

where under the small angle approximation

$$
|\mathbf{u}| \theta_{u}=\Delta u_{x} .
$$

If we assume that $\mathrm{NA} \approx \theta_{u}$ and $|\mathbf{u}| \approx 1 / \lambda$, the spatial resolution is equal to

$$
\Delta_{x}=\frac{\lambda}{\mathrm{NA}} .
$$

Similarly, from the wave vector geometry, the spatial frequency resolution along $z\left(\Delta u_{z}\right)$ is determined by

$$
\Delta u_{z}=\Delta u_{z, \max }-\Delta u_{z, \min }=|\mathbf{u}|\left(1-\cos \left(\theta_{u}\right)\right)=|\mathbf{u}| \theta_{u}^{2} .
$$

Under the small angle approximation, the axial resolution is

$$
\Delta_{z}=\frac{\lambda}{\mathrm{NA}^{2}}
$$

After substituting the expression for NA from Eq. (16) into Eqs. (19) and (21), we see that lateral resolution is also defined as $\bar{\Delta}_{x} \approx 2 \Delta x_{o}$ and range resolution is defined as $\Delta_{z} \approx 4 \Delta x_{o}^{2} / \lambda$. Defining the lateral and axial resolution using NA describes resolution in terms of system geometry (a function of object distance), whereas the second metric is modeled as a function of feature size, $\Delta x_{0}$. The maximum of the two measures for lateral and axial resolution provides a baseline metric for resolution. We use these metrics for evaluating resolution from TV minimization object reconstructions in Section 5 .

This section provided motivation for implementing a Gabor geometry instead of an off-axis approach. The impact of detector sampling at the hologram plane was addressed and a relation between object sampling and frequency domain sampling was discussed. Finally, theoretical resolution metrics were derived.

\section{Reconstruction Methods and Simulations}

In this section, we discuss two reconstruction methods: 3D object estimation from a Gabor hologram and 3D object estimation from randomly subsampled Gabor holographic measurements. Subsampling is implemented to further analyze the impact of compressive measurement on 3D object estimation.

The continuous model for Gabor holography, modeled under the first Born approximation, is shown in Eq. (2). The detector plane is located at the $z_{h}=0$ plane in the $\mathbf{r}\left(x, y, z_{h}\right)$ coordinate system. The object data, $f$, are located in the $\mathbf{r}^{\prime}\left(x^{\prime}, y^{\prime}, z^{\prime}\right)$ coordinate system. The recorded hologram in Eq. (1) can be reformulated if we assume that $E_{r}\left(\mathbf{r}_{\mathbf{h}}\right)=1$ and if operations on $f_{o}\left(\mathbf{r}^{\prime}\right)$ in the convolution integral in Eq. (2) are expressed using an operator, $H$. After squared-reference field subtraction, we can represent the recorded hologram in algebraic notation using

$$
g=|H f|^{2}+H f+H^{*} f+n,
$$

where $g$ is an $N \times 1$ vectorized detector measurement, $H$ is a $2 D$ discrete system matrix, $f$ is an $M \times 1$ vectorized object representation $\left[f_{o}\left(\mathbf{r}^{\prime}\right)\right]$, and $n$ is the noise associated with the measurement. If we ignore the object-squared-field contribution in Eq. (22), we can establish a linear relationship between the detector measurement and object field distribution, $g=H f$.

Once we record a digital hologram, our goal is to estimate the object distribution, $f$. From [15], we know that the digitized holographic measurement is

$$
\begin{aligned}
g_{n_{1}, n_{2}} & =\sum_{l}^{-1} \mathbf{2 D} \\
& \times\left\{\hat{f}_{m_{1}, m_{2}, l} \exp \left(1 l \Delta_{z} \sqrt{\frac{1}{\lambda^{2}}-m_{1}^{2} \Delta_{u}^{2}-m_{2}^{2} \Delta_{u}^{2}}\right)\right\}_{n_{1}, n_{2}},
\end{aligned}
$$


where ${ }^{-1}$ represents the $2 \mathrm{D}$ inverse fast Fourier transform (FFT) operation, $\hat{f}$ represents the FFT of the object distribution, and the exponential term encompasses the transfer propagation function. Indices $n_{1}$ and $n_{2}$ are discrete indices for each spatial dimension $(x$ and $y), l$ is the discrete index for $z, m_{1}$ and $m_{2}$ are the Fourier-transformed indices, $\Delta_{z}$ is the resolution cell along the axial plane, and $\Delta_{u}$ is the sampling resolution in the Fourier domain due to discretization by the detector plane. Using Eq. (23), we can model $H$ in Eq. (22) as

$$
H=\frac{-1}{2 D} \mathbb{Q}_{2 D} .
$$

The adjoint system model of Eq. (23) is

$$
f=H^{\dagger} g,
$$

where $H^{\dagger}$ is the adjoint operator defined as

$$
H^{\dagger}={ }_{2 D} \mathbb{Q}_{2 D}^{\dagger-1} .
$$

Note that ${ }^{\dagger}$ represents the Hermitian transpose operation. The forward system matrix, $H$, models scattering/object field propagation via two FFTs and a quadratic phase term [24]. The FFT and inverse FFT operators are diagonal matrices, and the exponential term in Eq. (23) is expressed as a propagation quadratic phase matrix, $\mathbb{Q}=Q_{l, m_{1} \times m_{2}}$, where

$$
Q_{l=1, m_{1}, m_{2}}=\exp \left({ } l \Delta_{z} \sqrt{\frac{1}{\lambda^{2}}-m_{1}^{2} \Delta_{u}^{2}-m_{2}^{2} \Delta_{u}^{2}}\right) .
$$

We use the adjoint model in Eq. (25) for linear backpropagation object estimation.

Inverse propagation, otherwise known as backpropagation, is a linear method for 3D object field reconstruction. Figure 2(c) shows digitally backpropagated object fields at different $z$ planes. This result demonstrates the challenges associated with linear inverse propagation, as the undiffracted and outof-focus field contributions make object range detection challenging. Linear backpropagation provides an estimate for the 3D field and not the 3D object density. Minimizing the contribution of the undiffracted field or twin-image problem has been explored $[19,25]$. Conventional methods to increase range resolution require either multiple wavelengths or multiple projections [26,27]. By exploiting results from CS, we show it is possible to isolate objects along an axial plane where range resolution is consistent with the object's spatial extent using only a single $2 \mathrm{D}$ recording.

Recently, the TwIST 2D TV minimization algorithm was adapted for 3D tomographic estimation from a single digitized 2D hologram [15,28]. Forward and adjoint operators in Eq. (24) and (25) are incorporated into the TwIST algorithm. Analytical rigor associated with the algorithm has previously been formulated [15]. The algorithm minimizes a convex quadratic problem with the addition of a sparsity constraint. The sparsity constraint is enforced on the gradient [see Eq. (30)] of the object estimate, $f$. Applying the constraint enables improved 3D tomographic estimation from a $2 \mathrm{D}$ measurement [29] because the twin-image problem associated with the inverse propagation method is reduced. Even though we neglect the effect of the nonlinear term $|H[f]|^{2}$ in the system model shown in Eq. (22), a new term, $e$, is added to model measurement error. The final measurement model becomes

$$
g=2 \mathfrak{R}\{H[f]\}+n+e .
$$

In this paper, we minimize a convex quadratic function using TV regularization denoted by

$$
f^{*}=\underset{f}{\operatorname{argmin}}\|g-H f\|_{2}^{2}+\tau \Phi_{T V}(f),
$$

where $f^{*}$ is the $3 \mathrm{D}$ object estimate and $\tau$ is the regularization constant. The $\Phi_{T V}$ function is defined as

$$
\Phi_{T V}=\sum_{l} \sum_{i, j} \sqrt{\left(f_{i+1, j, l}-f_{i, j, l}\right)^{2}+\left(f_{i, j+1, l}-f_{i, j, l}\right)^{2}},
$$

where $l$ represents the discrete index in $z$ and $i$ and $j$ correspond to discrete indices for a $2 \mathrm{D}(x, y)$ spatial extent. The $\Phi_{T V}$ function preserves edges while imposing smoothness on a solution. The regularization constant, $\tau$, determines the convergence and image quality of the estimate. The TV-regularization algorithm in Eq. (29) is considered as a special case in a Bayesian framework because TV regularization is similar to determining a maximum a posteriori estimate with a TV-prior distribution. Again, TV minimization reconstructions enable twin-image reduction and squared-field reduction otherwise dominant in linear inverse propagation. Figure 3 shows TV minimization object reconstructions. Undiffracted energy contributions are localized at the $z=0$ plane, while twin-image contributions are weakly scattered to the object field planes and primarily located at the conjugate reconstructed object planes [i.e., $z \leq 0$ in Fig. 3]. Compared to the inverse propagation results in Fig. 3, Fig. 3 demonstrates twin-image suppression and squared-field suppression because TV-imposed sparsity enables estimation of the object density and not the field distribution.

In this paper, we also analyze the impact of subsampled holographic measurements on object reconstruction. We apply a binary-valued, pseudorandom transmittance function, $t(x, y)$, to reduce the number of measurements at the hologram plane. The transmittance function is defined as

$$
t(x, y)=\sum_{p, q} t_{p, q} \operatorname{rect}\left[\frac{x-p \Delta_{T}}{\Delta_{T}}, \frac{y-q \Delta_{T}}{\Delta_{T}}\right] .
$$




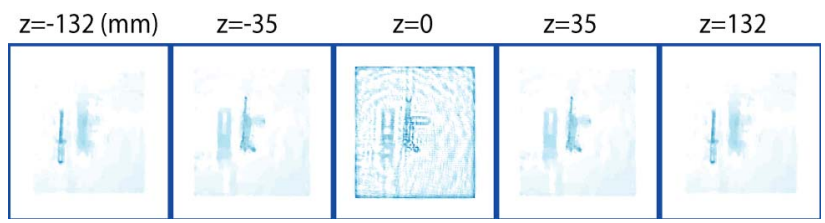

Fig. 3. (Color online) Transverse slices from TV minimization reconstructions at different $z$ planes. A dominant squared-field term is confined to the $z=0$ plane.

The pseudorandom measurement matrix contains transmissive (1) and opaque (0) openings. We assume that the sampling pitch, $\Delta_{T}$, of the matrix is equal to the receiver sampling pitch. Similarly, indices for the discrete representation of the measurement matrix and detector matrix are equivalent $\left(p=n_{1}\right.$ and $\left.q=n_{2}\right)$. The sparse detector measurement, $\breve{g}$, is defined as

$$
\breve{g}_{n_{1}, n_{2}}=t_{n_{1}, n_{2}} g_{n_{1}, n_{2}} .
$$

If the discrete transmittance function, $t_{n_{1}, n_{2}}$, for the aperture is represented as a diagonal matrix, $W$, then the new forward and adjoint models are represented as

$$
g=W F_{2 D}^{-1} Q F_{2 D} f
$$

and

$$
f=F_{2 D}^{\dagger} Q^{\dagger}\left[F_{2 D}^{-1}\right]^{\dagger} W^{\dagger} g .
$$

The TV minimization algorithm is adapted for hologram measurement subsampling using the aforementioned forward and adjoint models. Sparse detector measurements are motivated by an existing need to reduce scan times in MMW imaging applications. Simulations incorporating the above system model are explored in the next section to study the impact of a subsampled 2D holographic measurement on 3D tomographic reconstruction. The goal is to show that a reduction in the number of measurements can be achieved without a huge compromise in $3 \mathrm{D}$ object reconstruction.

\section{A. Simulation Results}

In simulation, we demonstrate $3 \mathrm{D}$ object estimation from $2 \mathrm{D}$ digitized holographic measurements, as well as quantify the impact of sparse holographic measurement on 3D object estimation. In this section, our simulated detector measurements are constructed using Eq. (22). Our sparse measurement model is based on Eq. (33) and incorporated into Eq. (22). Recall that for sparse measurement, we apply a pseudorandom transmittance function to holographic measurements. Examples of the sampling matrices under test are shown in Fig. 4 . We evaluate reconstructions from simulated detector measurements corrupted and uncorrupted by additive white Gaussian noise (AWGN). Noise is added to simulated detector measurements using the MATLAB "awgn" command, where the measurement SNR is specified. Also, we analyze the impact of $0 \%$ to $54.68 \% 2 \mathrm{D}$ measurement reduction on 3D object estimation. For 3D object estimation, we compare two inversion methods: a linear backpropagation method and nonlinear TV minimization. We quantify reconstruction efficacy using peak signalto-noise ratio (PSNR). We define PSNR as

$$
\begin{aligned}
\operatorname{PSNR}(d B) & \stackrel{\text { def }}{=} 20 \log _{10} \\
& \times\left\{\frac{\operatorname{MAX}_{A}}{\frac{1}{n_{x} n_{y} d} \sum_{i=1}^{n_{x}} \sum_{j=1}^{n_{y}} \sum_{l=1}^{d}\left[A_{i, j, l}-B_{i, j, l}\right]^{2}}\right\},
\end{aligned}
$$

where $A$ represents the synthetic object, $B$ represents the object estimate, $n_{x}$ and $n_{y}$ represent the number of detector pixels along each spatial dimension, $d$ represents the number of axial planes, and PSNR units are in decibels $(\mathrm{dB})$. As expected, PSNR decreases as the percentage of samples removed increases. Also, the addition of AWGN decreases PSNR.

Simulation system parameters mimic that of our experimental platform. Each simulated hologram measures $128 \times 128$ pixels with a pixel pitch of $2.32 \mathrm{~mm}$. Our synthetic objects were modulated by a $94 \mathrm{GHz}$ reference illumination field. We tested two 3D objects: synthetic slits and a synthetic gun and dagger object located at different depths along the axial plane. We used the axial resolution definition in Subsection 2.B as the metric for object separation along the axial plane. The simulated synthetic slit targets follow this convention. For example, the smallest object feature size of the synthetic slit target measures one wavelength $(\approx 3 \mathrm{~mm})$ and the object distance from the detector measures $20 \mathrm{~mm}$, which results in an object separation distance of approximately $10 \mathrm{~mm}$. The synthetic gun and dagger target in this section attempts to mimic experimental measurements detailed in Section 5. Further, TV minimization reconstruction depths are based on the predetermined object locations. Note that the synthetic targets are 2D and have a uniform amplitude. Real objects explored in Section 5, however, are 3D and located in multiple planes. The impact of $3 \mathrm{D}$

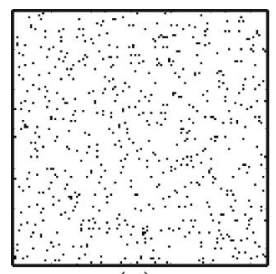

(a)

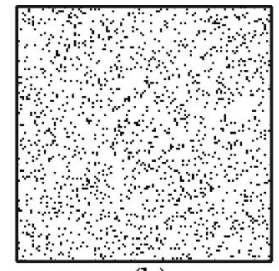

(b)



(c)

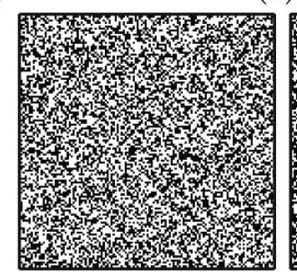

(d)

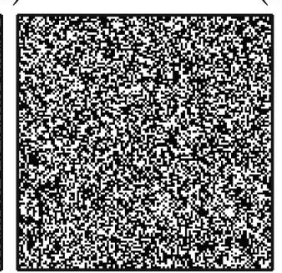

(e)
Fig. 4. Sampling windows for sparse measurement where (a) $3.9 \%$, (b) $9.77 \%$, (c) $23.83 \%$, (d) $44.56 \%$, and (e) $54.68 \%$ of the measurements are removed. 
spatially extended objects along multiple axial planes is not investigated in this manuscript.

First, we simulated a digital hologram of a uniform $3 \mathrm{D}$ slit object using Eq. (22). Row one, row two, and row three of the slit object were located in three separate planes: $20 \mathrm{~mm}, 30 \mathrm{~mm}$, and $40 \mathrm{~mm}$ away from the detector plane. The slits are 21 pixels long. Row one contained three sets of three-pixel-wide slits. Slit pairs were separated by one, two, and three pixels, respectively. Row two contained three sets of five-pixel-wide slits. Slit pairs were separated by two, four, and one pixel(s), respectively. Row three consisted of three sets of two-pixel-wide slits. Slit pairs were separated by one, two, and four pixels, respectively. In the postdetection process, linear backpropagation and TV minimization reconstructions are compared and shown in Fig. 5. Note that the reconstructions in Fig. 5 are from simulated detector measurements corrupted by AWGN at a $30 \mathrm{~dB}$ measurement SNR. Backpropagation accurately estimates the object wave field, while TV minimization accurately estimates the object spatial extent along the axial plane. Improved twin-image suppression is obtained with TV minimization. Note that the slit object addresses spatial resolution limitations as fewer holographic measurements are used for data inversion. These limitations are object size-dependent. A set of five-pixel-wide $(11.6 \mathrm{~mm})$ slits separated by four pixels $(9.23 \mathrm{~mm})$ are resolved after nonlinear inversion, even though $44.46 \%$ of the holographic measurements are removed. Linear backpropagation object field estimation is challenging when $54.68 \%$ of the holographic measurements are removed. A closer inspection of Fig. $\underline{5}$ demonstrates other spatial resolution limitations based on smaller object feature sizes.

Second, we simulated a hologram of a 3D synthetic object where a uniform amplitude 2D gun and a 2D dagger were placed at different distances along the axial plane. The synthetic data was modeled after experimental data in Fig. 12. The synthetic gun and dagger were located $30 \mathrm{~mm}$ and $140 \mathrm{~mm}$ away from the hologram plane. The smallest feature on the gun, located at the barrel, measures two pixels $(4.64 \mathrm{~mm})$. The smallest feature on the dagger, located at the edge of the blade, measures four pixels $(9.28 \mathrm{~mm})$. Figure 6 presents linear and nonlinear inversion estimates of the holographically measured data corrupted by AWGN at a $30 \mathrm{~dB}$ measurement SNR. While linear backpropagation fails to estimate the object field both spatially and longitudinally with $54.68 \%$ of the measurements missing, TV minimization succeeds in providing adequate spatial resolution and object localization along the axial plane.

While Figs. $\underline{5}$ and $\underline{6}$ only consider reconstructions from 3D synthetic targets corrupted by AWGN at a single measurement SNR, Fig. $\underline{7}$ quantitatively summarizes the effect of AWGN using different sampling strategies. Simulation results in Fig. 7 use various values of $\tau(0.2-1)$. The number of iterations, however, was fixed. Values for $\tau$ were chosen by trial and error to visually produce the best object contrast in the reconstruction estimate. The number of iterations was chosen such that the relative difference in the

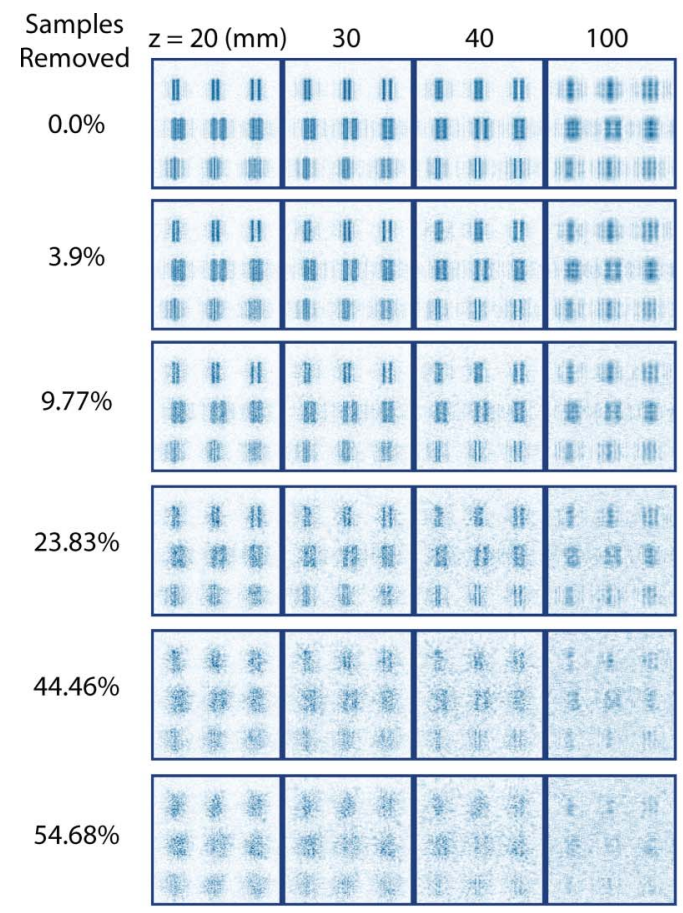

(a)
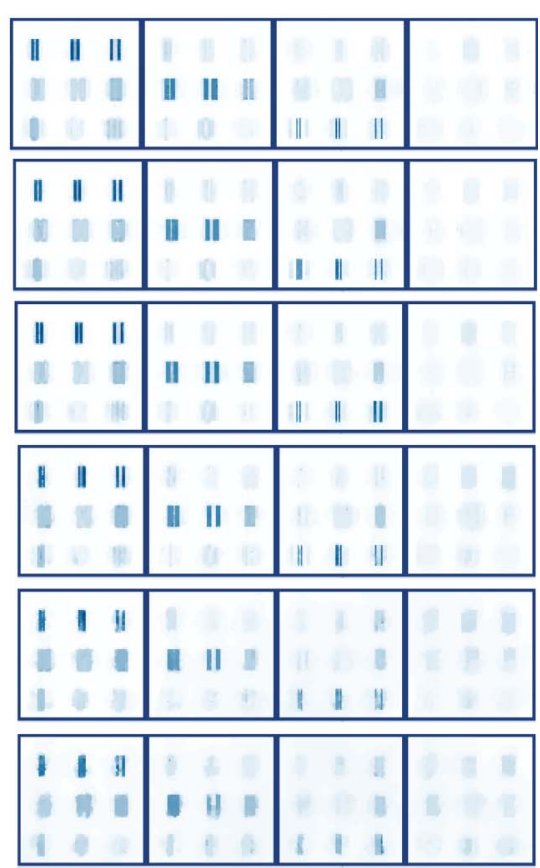

(b)

Fig. 5. (Color online) Synthetic 3D slit object results with an applied transmittance function and corrupted by AWGN at a $30 \mathrm{~dB}$ measurement SNR using (a) backpropagation and (b) TV minimization for 3D tomographic object estimation. Various values for $\tau(0.2-1.0)$ are used for sparsely sampled $(0.0-54.68 \%)$ TV reconstructions (see Table $\underline{1}$ ). 


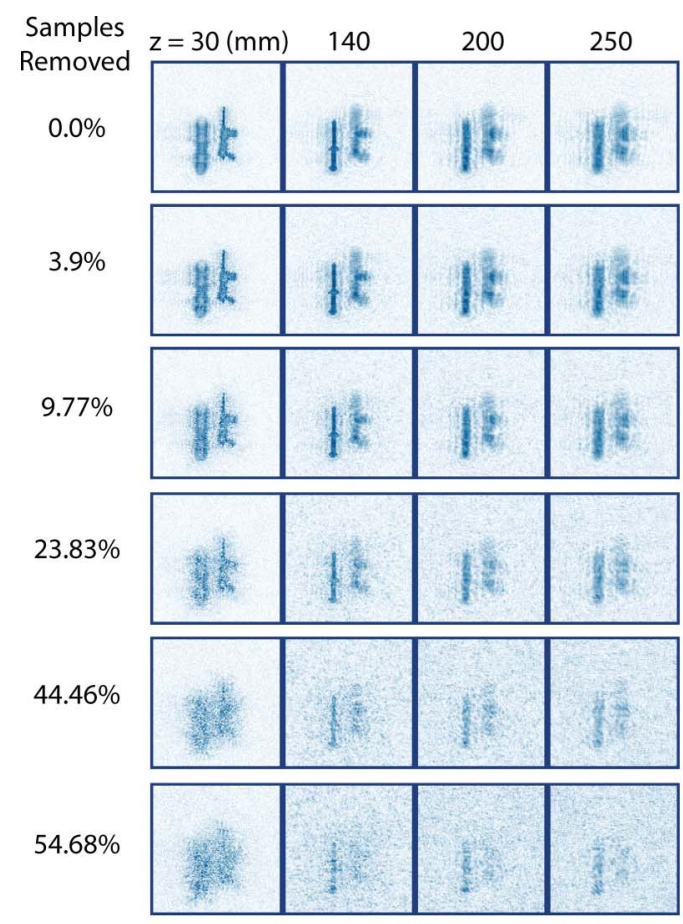

(a)

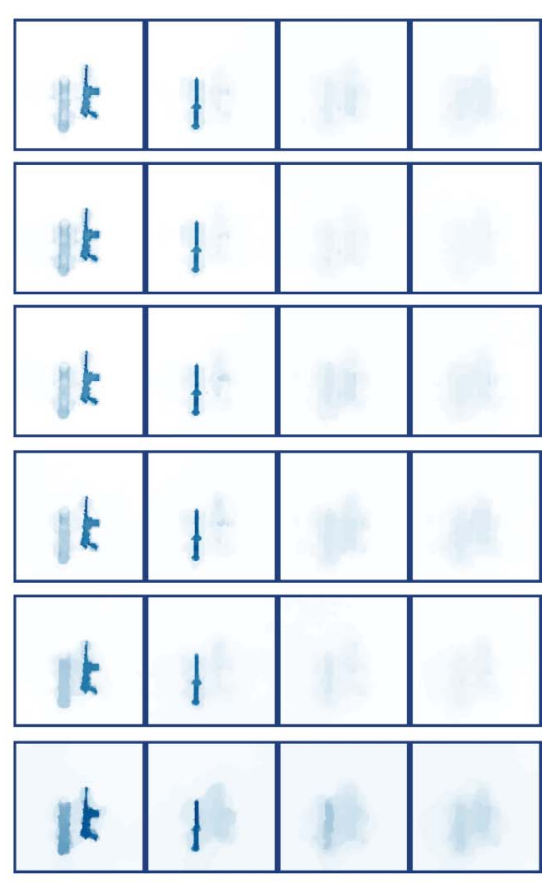

(b)

Fig. 6. (Color online) Synthetic 3D dagger and gun object results with an applied transmittance function and corrupted by AWGN at a $30 \mathrm{~dB}$ measurement SNR using (a) backpropagation and (b) TV minimization for $3 \mathrm{D}$ tomographic object estimation. A $\tau$ value of 0.2 is used for TV minimization reconstructions from sparsely sampled detector measurements corrupted by AWGN (see Table 1).

objective function was nominal. In Figs. 7 and 13 , different measurement SNR noise levels were analyzed. Using the same algorithm parameters, multiple reconstruction estimates at each noise level were consistent. The TV minimization reconstruction of the synthetic 3D slit target for all sampling schemes converged at a $30 \mathrm{~dB}$ measurement SNR with a PSNR range between $25 \mathrm{~dB}$ and $30 \mathrm{~dB}$. The results in Fig. 7(a) show that a $54.7 \%$ measurement reduction only incurs a $5 \mathrm{~dB}$ loss in PSNR. In comparison, the synthetic 3D gun and dagger object converges around a $40 \mathrm{~dB}$ measurement SNR, which yields approximately a $32 \mathrm{~dB}$ PSNR. For the synthetic dagger and gun object case, measurement reduction between $0 \%$ and $54.7 \%$ provides a PSNR range between $29 \mathrm{~dB}$ and $32 \mathrm{~dB}$. Because the 3D gun and dagger gradient sparsity is smaller, it is not surprising that TV minimization results yield a higher PSNR than the $3 \mathrm{D}$ slit target. Lastly, backpropagation results for both 3D synthetic objects in Figs. 7(c) and 7(d) converge at a $20 \mathrm{~dB}$ measurement SNR for all measurement schemes and yield a low reconstruction PSNR (17-20 dB).

In simulation, we can measure object sparsity by calculating the number of nonzero gradients for each $3 \mathrm{D}$ synthetic object under test. We measure object sparsity for each 3D object by calculating the gradient and totaling the nonzero values. The nonzero gradient, $\left|\nabla f_{i, j, l}\right|$, at the $(i, j)$ th pixel location in the $l$ th axial plane is calculated using

$$
\left|\nabla f_{i, j, l}\right|=\sqrt{\left(f_{i+1, j, l}-f_{i, j, l}\right)^{2}+\left(f_{i, j+1, l}-f_{i, j, l}\right)^{2}}
$$

Note that larger 3D objects produce more nonzero gradients. The nonzero gradients calculated for the synthetic 3D slit object and the synthetic 3D dagger and gun object were 2580 and 1129 . Recall that the simulated 2D hologram measures $128 \times 128$ pixels. We analyze the ratio between the total number of measurements $(N)$ recorded at the detector plane to the object sparsity/nonzero gradients calculated. This ratio is listed in Table 1 as the sparsity ratio. In $\mathrm{CS}$, the number of measurements required for adequate signal estimation is $N \geq S C \log (M)[12,15]$, where $S$ represents the number of nonzero gradients/ sparsity calculated in the gradient domain, $C$ represents an empirical constant value, and $M$ represents the original size of the vectorized object signal $f$. We can evaluate the reconstruction of the $3 \mathrm{D}$ slit object with a $54.68 \%$ holographic measurement reduction by analyzing the PSNR and sparsity ratio. A low PSNR is attributed to the low sparsity ratio shown in Table 1 . For example, a 3D synthetic gun and dagger object with a $54.68 \%$ measurement reduction yields a PSNR of 26.09 and has a sparsity ratio of 6.7. Because the sparsity ratio for the synthetic slit object with the same measurement reduction yields a low PSNR, we would need to record a larger number of holographic measurements to improve the reconstruction PSNR. While the synthetic 3D slit object addressed spatial resolution limitations and gradient domain object sparsity concerns in simulation, 


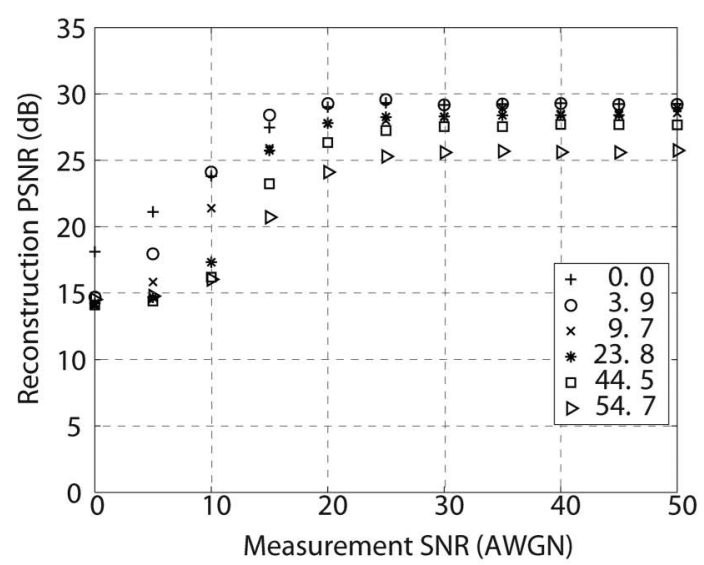

(a)

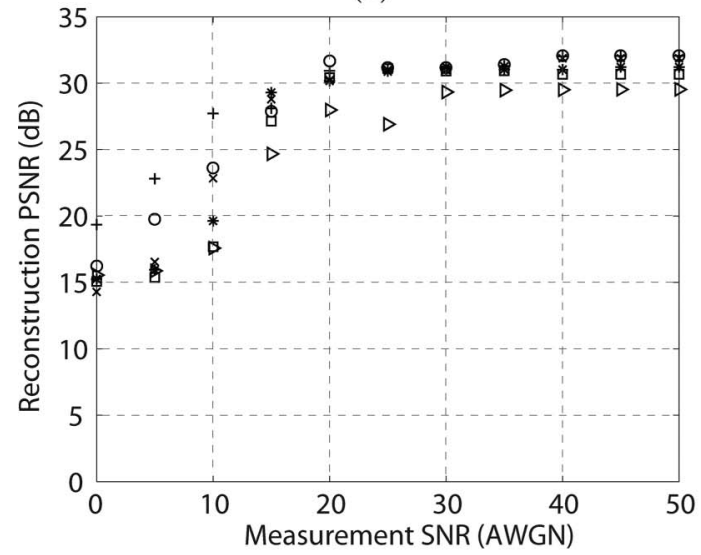

(b)

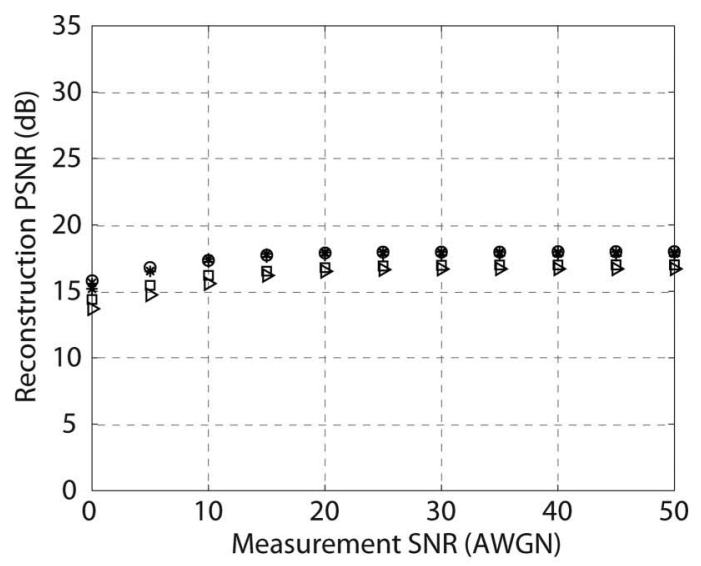

(c)

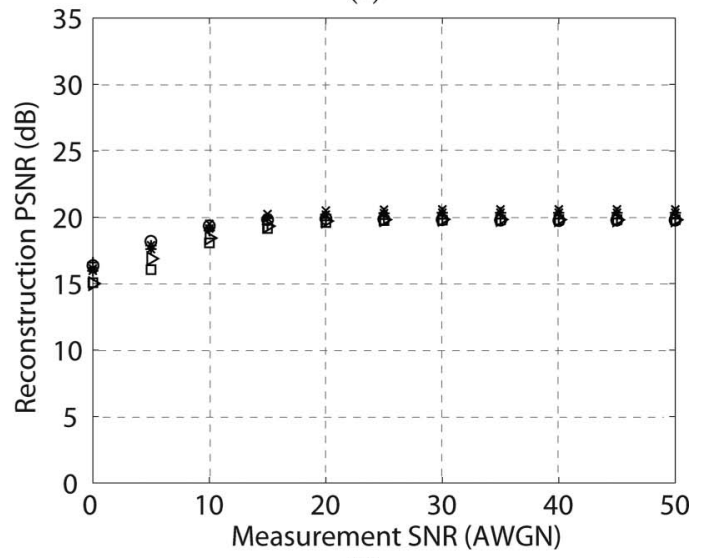

(d)

Fig. 7. Plot of reconstruction PSNR (in $\mathrm{dB}$ ) versus measurement SNR (in $\mathrm{dB}$ ) from MMW holography detector measurements corrupted by AWGN. TV minimization reconstruction results with 0.0-54.7\% measurement reduction are shown for the (a) synthetic slit target and (b) synthetic gun and dagger target. Backpropagation reconstruction results with $0.0-54.7 \%$ measurement reduction are shown for the (c) synthetic slit target and (d) synthetic gun and dagger target.

the synthetic 3D gun and dagger object is used to help evaluate experimental reconstructions taken of a semitransparent gun and dagger placed along an axial plane in Section $\underline{5}$.

\section{Experimental Setup}

Simulations in the previous section indicate that digital holography combined with a TV postdetection process is effective. To validate the simulations, we conducted various experiments measuring Gabor holograms. Our 2D hologram is recorded in a Gabor geometry, as shown in Fig. 8. RF energy is generated from a tunable InP Gunn diode oscillator and coupled to a WR-10 open waveguide with dimensions $2.54 \mathrm{~mm}(\mathrm{~W}) \times 1.27 \mathrm{~mm}(\mathrm{H})$. The oscillator is tuned

Table 1. Synthetic 3D Slit (ST) and 3D Gun and Dagger (GD) Sparsity

\begin{tabular}{cccc}
\hline & $\%$ Removed & ST Sparsity Ratio & GD Sparsity Ratio \\
\hline 1 & 0 & 6.35 & 14.51 \\
2 & 3.9 & 6.10 & 13.95 \\
3 & 9.77 & 5.73 & 13.09 \\
4 & 23.83 & 4.84 & 11.05 \\
5 & 44.46 & 3.53 & 8.06 \\
6 & 54.68 & 2.88 & 6.58 \\
\hline
\end{tabular}

to $94 \mathrm{GHz}$ and, after attenuation, is found to radiate with a peak power of $100 \mathrm{~mW}$. In the far field $\left(4 D^{2} / \lambda\right)$ of the waveguide aperture, a collimated reference beam is directed towards an object having spatial extent, $L_{x}$ in Fig. 8 . Actual reference beam dimensions $(B)$ are calculated using the illumination wavelength $(\lambda)$, waveguide aperture size $(D)$, and waveguide to object distance $\left[z_{1}\right.$ in Fig. 8] such that $B=$ $0.89 \lambda z / D[30]$. Energy diffracted from the object interferes with a reference field at a receiver plane where $N$ measurements are recorded with sampling pitch $d x$.

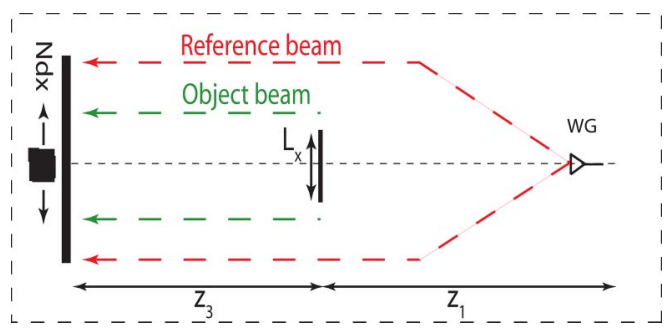

Fig. 8. (Color online) Optical schematic for MMW Gabor holography containing a waveguide (WG), object extent $\left(L_{x}\right)$, detector plane sampling with number of pixels $(N)$ and pixel pitch $(d x)$, waveguide to object distance $\left(z_{1}\right)$, and object to receiver distance $\left(z_{3}\right)$. 


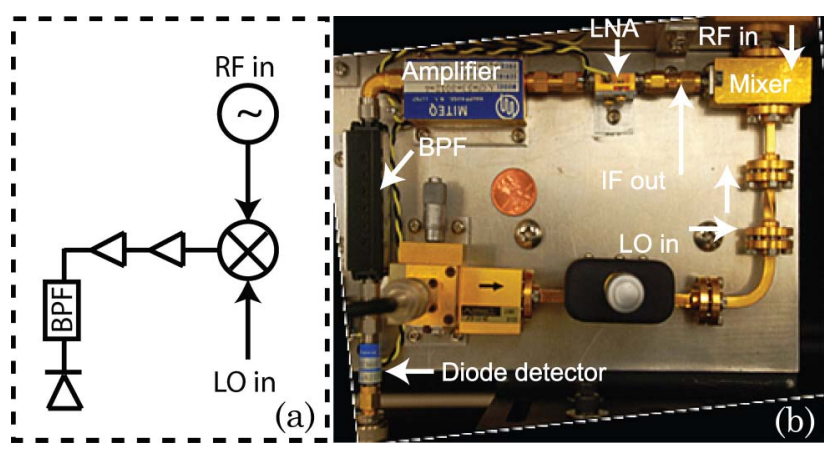

Fig. 9. (Color online) Superheterodyne receiver (a) circuit diagram and (b) experimental layout where incident energy ( $\mathrm{RF}$ in) is mixed with a local oscillator ( $\mathrm{LO}$ ), down converted to an intermediate frequency (IF), amplified by both an LNA and a second amplifier, filtered with a bandpass filter (BPF), and detected with a Schottky diode.

Our detector is an incoherent, single pixel receiver circuit shown in Fig. 9. Incident energy (at $94 \mathrm{GHz}$ ) at the receiver circuit is collected with a WR-08 open waveguide measuring $2.32 \mathrm{~mm}(\mathrm{~W}) \times 1.02 \mathrm{~mm}(\mathrm{H})$. In the circuit, energy is mixed with a local oscillator [LO in Fig. 9] at $85 \mathrm{GHz}$ and down converted to an intermediate frequency [IF in Fig. $9(\mathrm{~b})]$ at $9 \mathrm{GHz}$. The $I F$ signal is amplified twice with a low noise amplifier (LNA) and a MITEQ (8-12 GHz) amplifier. A bandpass filter is applied to the signal before a voltage measurement is read from a Schottky diode detector. The bandpass filter passes frequencies between $7-11 \mathrm{GHz}$, thereby making the receiver spectrally sensitive over a bandwidth of $4 \mathrm{GHz}$.

The output of the diode is a DC voltage, and measurements recorded with the receiver circuit confirmed that the system was operating in the linear region. We measured the sensitivity of the receiver as $48.31 \mathrm{mV} / \mu \mathrm{W}$. Also, there exists a DC offset in the receiver response due to the inherent circuit noise, as well as the data acquisition system. We calculate this DC offset or mean noise value by recording 1000 measurements per pixel over a region of $60 \times 60$ pixels. The mean background/noise value is $15 \mathrm{mV}$. The standard deviation of the noise level about the mean $(0.1472 \mathrm{mV})$ provided an indication

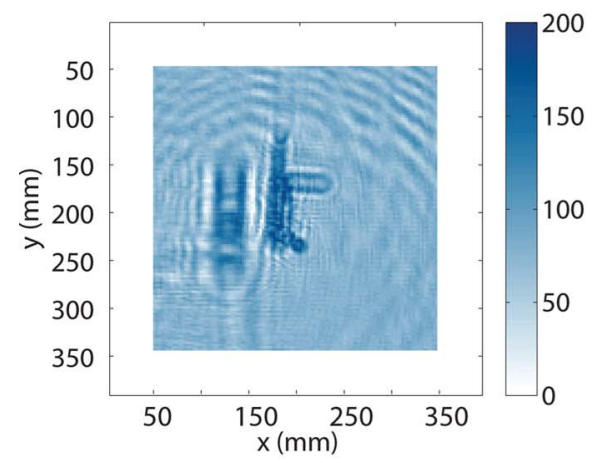

(a)

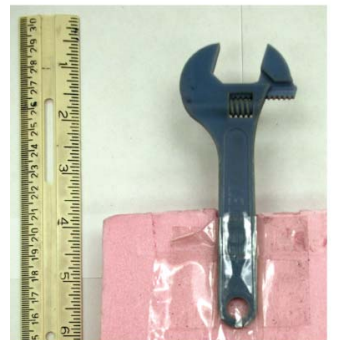

(a)

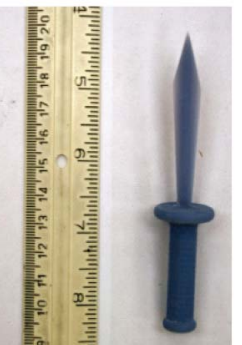

(b)

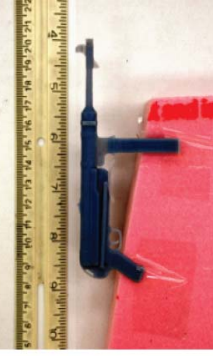

(c)
Fig. 10. (Color online) Object scale of a semitransparent polymer (a) wrench, (b) dagger, and (c) gun.

for the circuit noise caused by the receiver. Also, while the mean signal value within the recorded composite hologram is object dependent, the mean signal value for the reference beam is greater than 20 times the mean background signal. Thus, our composite holograms were not limited by inherent circuit noise or background noise levels.

For real-time measurement, we acquired voltages from our receiver circuit using a National Instruments 9(NI) USB high-speed M series data acquisition module (USB-6251). A LabView GUI was used to automate the data collection process from the NI-DAQ module, as well as to automate dual-axis $(x-$ $y$ ) translation of the receiver circuit. For receiver translation, we used a step size sampling rate of 1000 samples per second. Our sampling process involved scanning a step size of one-fifth of a $2.32 \mathrm{~mm}$ pixel pitch (step size of 73 motor steps) in the horizontal direction to more accurately acquire data using a raster-scan method. In the vertical direction, our step size equaled a $2.32 \mathrm{~mm}$ pixel pitch (step size of 365 motor steps). Further, we averaged 21 samples per step in both the horizontal and vertical directions. For all holograms detailed in this paper, we record a raster-scanned $(x-y) 128(\mathrm{H}) \times 640(\mathrm{~W}) \mathrm{im}$ age. The data acquisition time for a raster-scanned $128 \times 640$ pixel image took about 28 minutes.

\section{Experiment Results}

We recorded several Gabor holograms using the incoherent receiver circuit described above. We bin



(b)

Fig. 11. (Color online) Experimental holographic recording of a (a) model dagger and a model gun and (b) model dagger, model gun, and model wrench located in different $z$ planes. 
our raster-scanned image by five along the horizontal direction to generate a $128 \times 128$ pixel image. Each hologram is digitally recorded with 16 bit accuracy, as specified by the DAQ module. In the postdetection process, the composite hologram is reference beam subtracted, DC filtered in the spectrum of the hologram, and zero padded to create a $168 \times 168$ pixel image. Zero padding the system matrix helps to avoid circular convolution effects from the FFT operation used in the forward and adjoint models in the TV minimization algorithm. In this section, we compare two methods for 3D object reconstruction: conventional backpropagation and TV minimization algorithms detailed in Section 3. Recall that Fig. 3 depicts a squared-field term isolated at the $z=\overline{0}$ plane and twin-images located at object planes $z \leq$ 0 using the TV minimization algorithm. For simplicity, we compare and evaluate both the backpropagated and TV minimization reconstructions at $z>0$ planes.

Figure 10 illustrates three semitransparent polymer objects analyzed in this paper. Specific

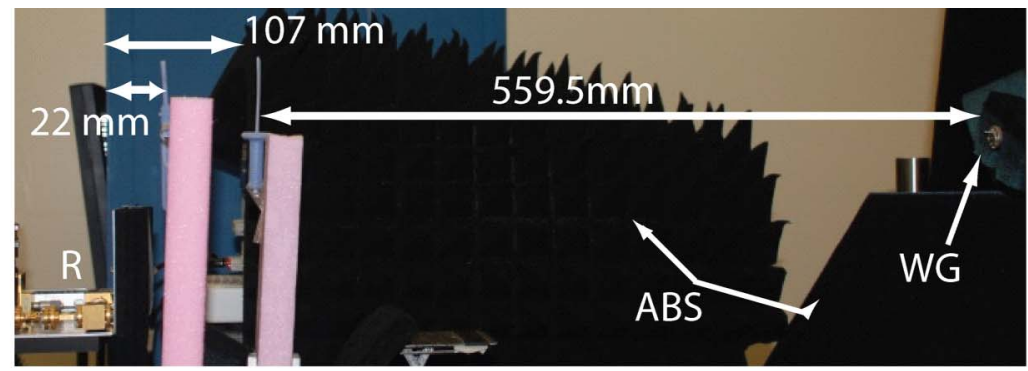

(a)

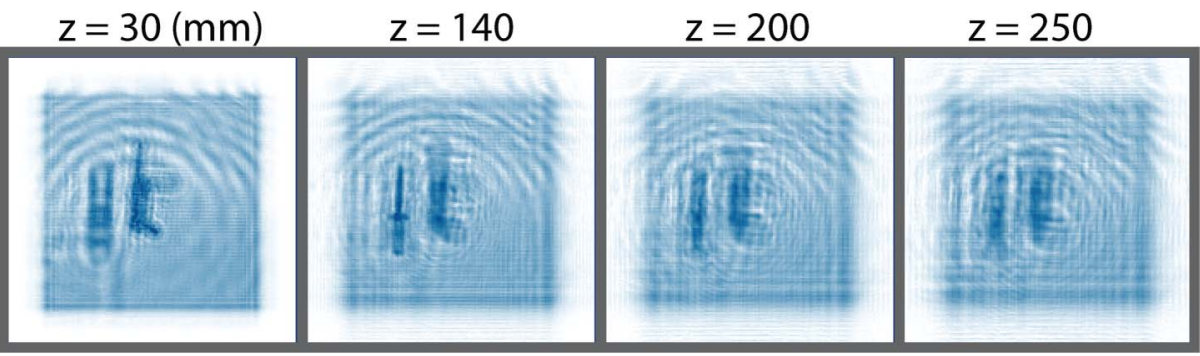

(b)

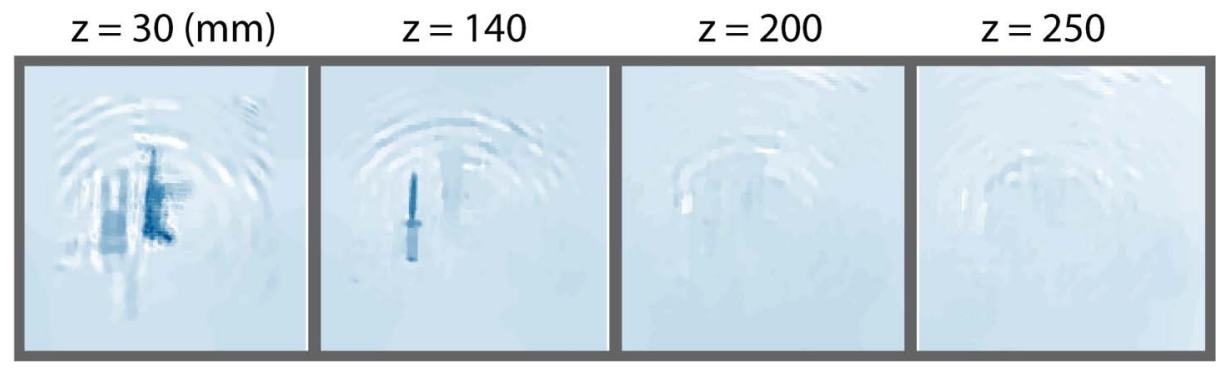

(c)

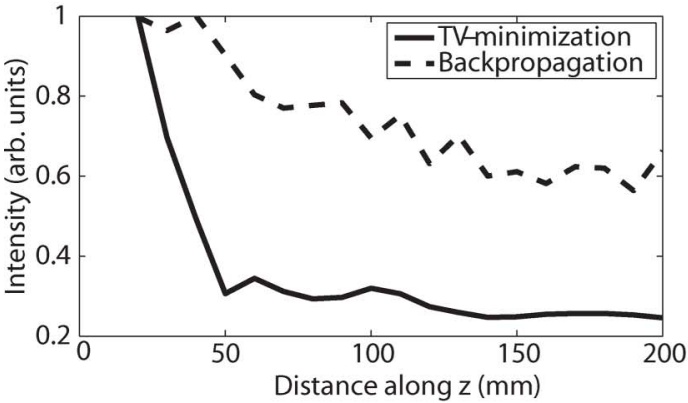

(d)

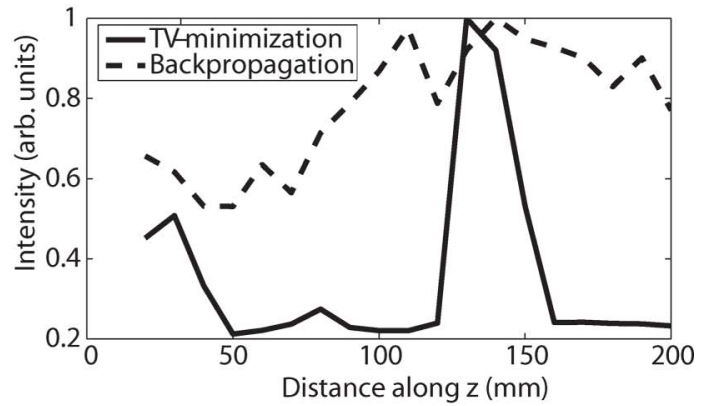

(e)

Fig. 12. (Color online) Experiment with a polymer model gun and dagger placed at two different distances along the axial plane. (a) Photograph of the experiment. Transverse slices in four different $z$ planes of the (b) backpropagated and (c) TV minimization reconstructions. Amplitude pixel $(x, y)$ as a function of $z$, in $10 \mathrm{~mm}$ increments, where TV minimization and backpropagation for a central point on the (d) barrel of the gun and (e) on the blade edge of the dagger are plotted. 


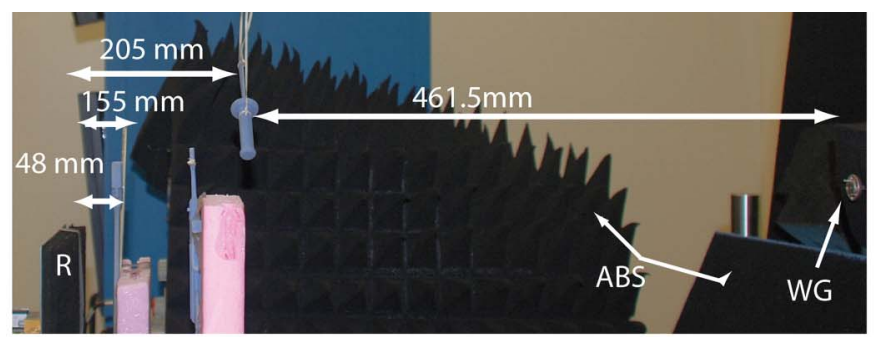

(a)

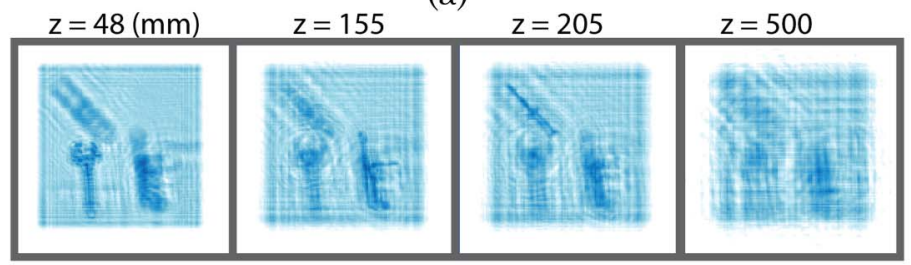

(b)

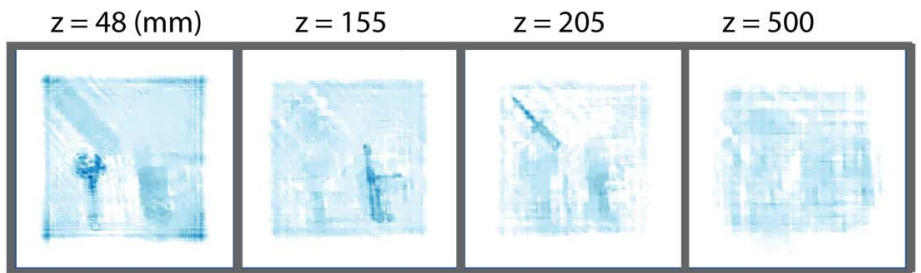

(c)

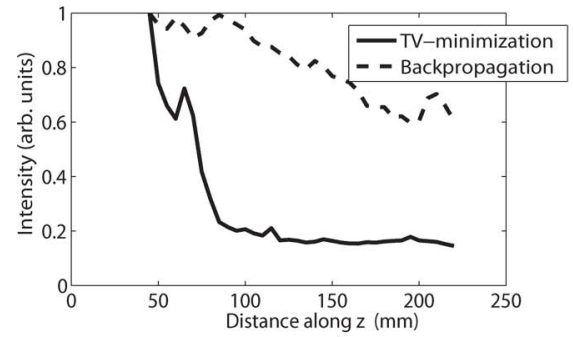

(d)

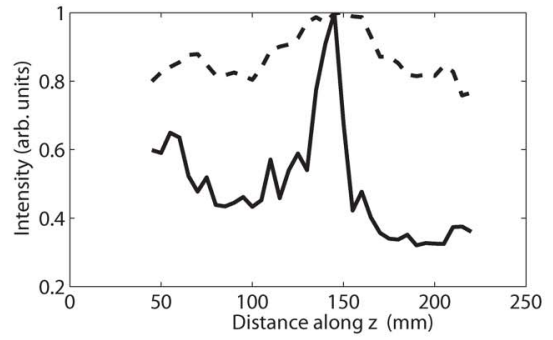

(e)

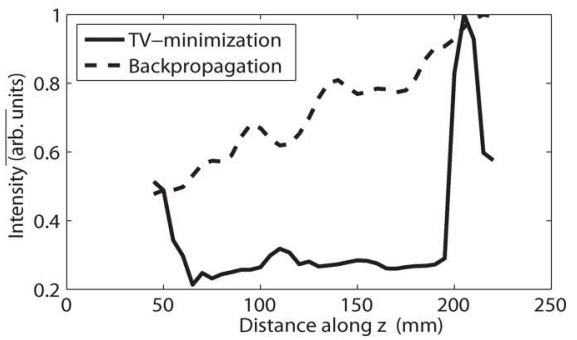

(f)

Fig. 13. (Color online) Experiment with a polymer model wrench, gun, and dagger placed at three different distances along the axial plane. (a) Photograph of the experiment. Transverse slices in four different $z$ planes of the (b) backpropagated and (c) TV minimization reconstructions. Amplitude pixel $(x, y)$ as a function of $z$, in $5 \mathrm{~mm}$ increments, where TV minimization reconstructions and backpropagation estimates for a center point on the (d) wrench, (e) gun, and (f) dagger are plotted.

properties associated with the object polymer material have been addressed elsewhere [31]. These objects were made with an Eden $33 \overline{3}$ prototyping machine that lays thin layers of photopolymer and UV cures each layer, building a $3 \mathrm{D}$ object slice by slice. We uniformly illuminated a $5 \mathrm{~mm}$ thick sheet of the polymer material under test [see Fig. 10] with $94 \mathrm{GHz}$ energy. The material was found to attenuate the incident energy by less than 20\%. In the experiment, we mounted the polymer objects to Styrofoam blocks (also semitransparent in the MMW) or suspended the objects using string. Test objects, in Fig. 10, include a model wrench with 2 in $(\mathrm{W}) \times 6$ in $(\mathrm{H})$ spatial extent, a model dagger with $0.25-0.5 \mathrm{in}(\mathrm{W}) \times 14 \mathrm{in}(\mathrm{H})$ spatial extent, and a model gun with $0.16-1$ in $(\mathrm{W}) \times 5$ in $(\mathrm{H})$ spatial extent. We used combinations of these test objects in the experimental setup. Further, we used MMW $\mathrm{RF}$ absorber [ABS in Figs. 12(a) and 13(a)] to minimize stray reflected energy collected at the receiver.
We conducted three separate experiments. In each experiment, the spatial extent of the $128 \times 128$ pixel hologram was $296.96 \mathrm{~mm}$ in both the horizontal and vertical dimensions. The holograms were zero padded to $168 \times 168$ pixels. Zero padding the hologram does not have any impact on reconstruction because we can assume that no object data exist in those regions. In the first experiment [see Fig. 12(a)], we recorded a hologram of a polymer model gun and model dagger placed $22 \mathrm{~mm}$ and $107 \mathrm{~mm}$ away from the receiver platform, as shown in Fig. 11(a). Recall that the optical schematic for the hologram recording geometry is shown in Fig. 8. Using linear inversion, we backpropagate the holographic measurement to four different $z$ planes, as shown in Fig. 12(b). We see that linear backpropagation estimates the 3D electromagnetic object field at each $z$ plane, but out-of-focus diffracted energy present in all $z$ planes hinders object localization. In Fig. 12(c), however, TV minimization results provide a means for localizing 




(a)



(c)
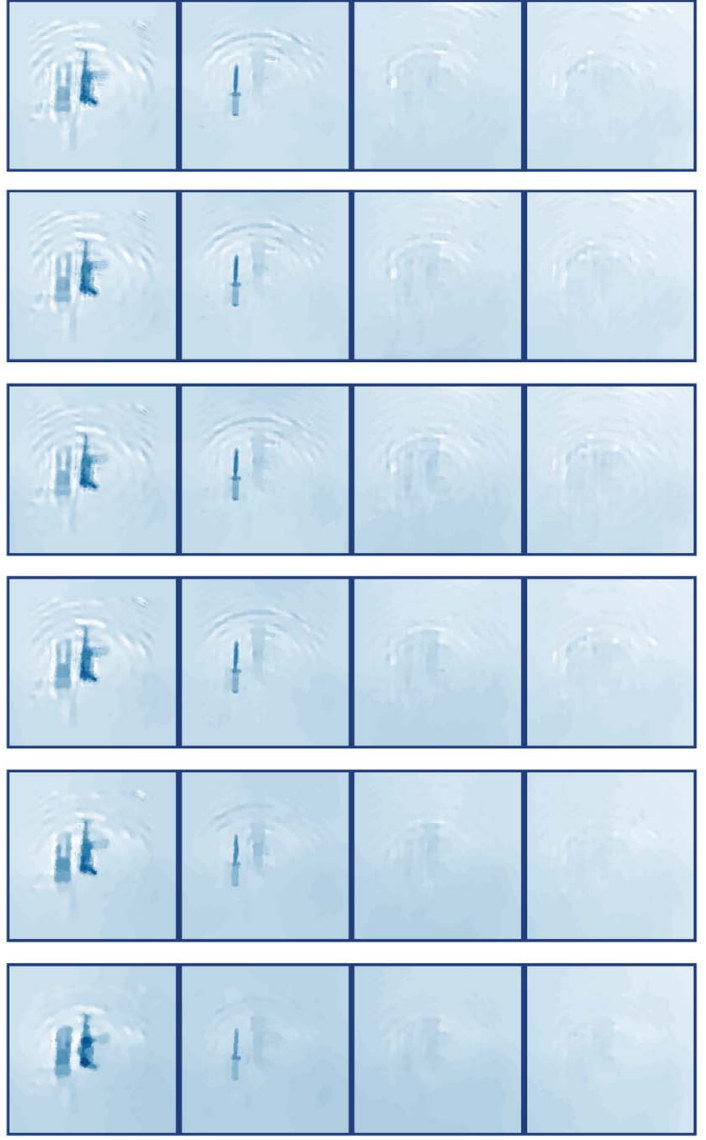

(b)



(d)

Fig. 14. (Color online) Sparse measurement reconstruction of experimental data using (a) linear backpropagation and (b) TV minimization for 3D object estimation. Amplitude of a central pixel $(x, y)$ on the blade edge of the dagger as a function of $z$, plotted in $10 \mathrm{~nm}$ increments, from (c) 3.9\% holographic measurement removal and (d) $54.68 \%$ holographic measurement removal.

objects to their corresponding $z$ planes. Note that the baseline axial resolution metrics, derived in Section 2, suggest an axial resolution of approximately $20 \mathrm{~mm}$ for an object feature size of $4 \mathrm{~mm}$. The pixel amplitude as a function of distance in $z$ is plotted in $10 \mathrm{~mm}$ increments in Figs. 12(d) and 12(e). These plots are generated at a finer resolution than the theoretical limit at $20 \mathrm{~mm}$. We plot the pixel amplitude at a central pixel $(x, y)$ located at the barrel of the synthetic gun and at the blade edge of the synthetic dagger. The axial plots in Figs. 12(d) and 12(e) show that TV minimization reconstruc- tions falls short of the theoretical limit. While backpropagation results provide an estimate of the object field along the axial plane, TV minimization enables object localization that is consistent with the spatial extent of the object. Our experimental axial resolution with the gun and dagger experiment is approximately $30 \mathrm{~mm}$. Errors are attributed to the residual diffracted energy (out-of-focus energy) found in neighboring $z$ planes for the TV minimization results. We can minimize this measurement error by acquiring a larger number of measurements $(>128$ pixels). 
In a second experiment, we placed a semitransparent polymer wrench, gun, and dagger at three different distances away from the receiver [see Fig. 13(a)]. These distances were $48 \mathrm{~mm}, 155 \mathrm{~mm}$, and $20 \overline{\mathrm{mm}}$. We recorded a digital hologram of the three objects, as shown in Fig. 11(b). A higher background signal, compared to the previous experiment, is attributed to an increase in illumination source power. We estimate the 3D object using linear backpropagation and TV minimization. Object estimates are reconstructed at four separate $z$ planes, as depicted in Figs. 13(b) and 13(c). Linear backpropagation estimates reveal object field distributions in each $z$ plane and limited object localization, while TV minimization estimates show a reduction in out-of-focus contributions from twin images. Amplitude data from a single spatial location in each object is plotted in increments of $5 \mathrm{~mm}$ along the axial plane in Figs. 13(d)-13(f). Object localization using TV minimization is shown in Figs. 13(c)-13(f), while backpropagation field estimates cannot provide any axial resolution. Still, the theoretical measure for a $4 \mathrm{~mm}$ feature size on the polymer gun object should enable $20 \mathrm{~mm}$ axial resolution. Axial plots from TV minimization reconstruction in Figs. 13(d) and 13(e) show an axial resolution of approximately $3 \overline{0 \mathrm{~mm}}$. Capturing more measurements with the receiver circuit would further improve TV estimation of the 3D object.

Lastly, we analyze the impact of sparse holographic measurements with real data. Sampling matrices shown in Fig. $\underline{4}$ are applied to experimental measurements. Because holographic measurements $(128 \times$ 128 pixels) are zero padded to a spatial extent of $168 \times$ 168 pixels, we zero pad the $128 \times 128$ transmittance functions, discussed in Section 2 , to the measurement data size. For backpropagation estimates, the detector pixel locations containing absolute zero values (due to the subsampling measurement matrix) were substituted with interpolated values calculated from measurements at neighboring pixels. Similar to the synthetic gun and dagger simulations in Section 2, experimental data show adequate spatial resolution and object localization along the axial plane when $54.68 \%$ of the measurement data are removed. This is feasible when a gradient sparsity constraint is used. Again, pixel amplitude versus distance in $z$ plots are generated in increments of $10 \mathrm{~mm}$ along the axial plane and shown in Figs. 14(c) and 14(d). Measurement reduction by $3.9 \%$ and $54.68 \%$ does not seem to impact object localization using TV minimization. However, we notice from simulation and experimental data that spatial resolution is sacrificed using backpropagation and TV minimization with a 54.68\% reduction of the measurement data.

In summary, this section discussed three experiments where objects were placed along the $z$ axis at either two different planes or three different planes. While data acquisition times were long (e.g., $<30$ minutes), 3D object estimation and improved object localization along the axial plane are made possible with TV minimization. Linear backpropagation accurately estimates the object field along the axial plane; however, out-of-focus diffracted fields hinder object localization. Sparse measurement impacts object spatial resolution using linear backpropagation because object field reconstruction is challenging. TV minimization results improve upon a linear backpropagation method where a twin-image term and a squared-field term affect object reconstruction. Sparse measurement reconstructions in simulation and with experimental data demonstrated that reconstructed object quality is maintained with fewer measurements. Also, sparse measurement reconstruction with experimental data further motivated the potential for decreasing data acquisition times using fewer scans.

\section{Summary and Conclusion}

An improved method for 3D tomographic estimation from 2D holographic measurements has been presented. Linear backpropagation estimation of a 3D object is hindered by a squared-field term and a twin-image term in reconstruction. TV minimization of 2D holographically recorded data shows suppression of these out-of-focus terms. Our nonlinear inversion scheme exploits object sparsity in the gradient domain. Further, simulations and experimental data reconstructions show that randomly sampled $2 \mathrm{D}$ holographic measurements enable 3D object estimation, even when $54.68 \%$ of the measurements have been removed. However, there is a degradation in spatial resolution. PSNR values in simulation decrease when holographic measurements are removed. Also, a sparse measurement scheme for adequate 3D object estimation is object dependent. Successful 3D object estimation from 2D holographic measurements using TV minimization depends on the object feature size $\left(\Delta x_{o}\right)$, which translates into the recorded hologram spatial extent.

This paper has focused on the analysis of semitransparent objects at millimeter wavelengths. In practice, objects of interest are not semitransparent. Thus, a reflection geometry may be better suited in a practical implementation. Data inversion techniques discussed in this paper can still be applied to a reflection geometry. The reflection case is more challenging, as speckle impacts holographic measurement. Also, because gradient-imposed sparsity is object dependent, a thorough object study on resolution for both transmissive and reflection cases still needs to be undertaken.

The authors would like to thank Ryoichi Horisaki, a member of the compressive holography team at Duke University, for his valuable suggestions. C. F. Cull acknowledges the support of a National Defense Science and Engineering Graduate Fellowship.

\section{References}

1. P. J. Costianes, "An overview of concealed weapons detection for homeland security," in Applied Imagery and Pattern Recognition Workshop, 2005 (IEEE, 2005), pp. 2-6.

2. R. McMillan, N. Currie, D. Ferris, Jr., and M. Wicks, "Concealed weapon detection using microwave and millimeter 
wave sensors," in Microwave and Millimeter Wave Technology Proceedings, 1998 (IEEE, 1998), pp. 1-4 .

3. D. McMakin, "Remote concealed weapons and explosive detection on people using millimeter-wave holography," in 30th Annual 1996 International Carnahan Conference on Security Technology, 1996 (IEEE, 1996) pp. 19-25.

4. M. R. Fetterman, J. Grata, G. Jubic, J. W. L. Kiser, and A. Visnansky, "Simulation, acquisition and analysis of passive millimeter-wave images in remote sensing applications," Opt. Express 16, 20503-20515 (2008).

5. S. Stanko, F. Kloppel, J. Huck, D. Notel, M. Hagelen, G. Briese, A. Gregor, S. Erukulla, H.-H. Fuchs, H. Essen, and A. Pagels, "Remote concealed weapon detection in millimeter-wave region: active and passive," Proc. SPIE 6396, 639606 (2006).

6. National Research Council Committee on Assessment of Security Technologies for Transportation, Assessment of Millimeter-Wave and Terahertz Technology for Detection and Identification of Concealed Explosives and Weapons (National Academies Press, 2007).

7. N. H. Farhat and W. R. Guard, "Millimeter wave holographic imaging of concealed weapons," in Proceedings of the Institute of Electrical and Electronics Engineers (IEEE, 1971), pp. 1383-1384.

8. D. Sheen, D. McMakin, and T. Hall, "Three-dimensional millimeter-wave imaging for concealed weapon detection," IEEE Trans. Microwave Theory Techn. 49, 1581-1592 (2001).

9. R. N. Anderton, R. Appleby, J. E. Beale, P. R. Coward, and S. Price, "Security scanning at $94 \mathrm{GHz}$," Proc. SPIE 6211, 62110C (2006).

10. R. Appleby, R. N. Anderton, N. H. Thomson, and J. W. Jack, "The design of a real-time $94 \mathrm{GHz}$ passive millimetre-wave imager for helicopter operations," Proc. SPIE 5619, 3846 (2004).

11. M. Kemp, "Millimetre wave and terahertz technology for detection of concealed threats-a review," in Infrared and Millimeter Waves, 2007 and the 2007 15th International Conference on Terahertz Electronics (IEEE, 2007), pp. 647-648.

12. E. Candes, J. Romberg, and T. Tao, "Stable signal recovery from incomplete and inaccurate measurements," Commun. Pure Appl. Math. 59, 1207-1223 (2006).

13. W. L. Chan, M. Moravec, R. Baraniuk, and D. Mittleman, "A single-pixel terahertz imaging system based on compressed sensing," Appl. Phys. Lett. 93, 121105 (2008).

14. W. L. Chan, M. L. Moravec, R. G. Baraniuk, and D. M. Mittleman, "Terahertz imaging with compressed sensing and phase retrieval," Opt. Lett. 33, 974-976 (2008).
15. D. J. Brady, K. Choi, D. L. Marks, R. Horisaki, and S. Lim, "Compressive holography,” Opt. Express 17, 13040-13049 (2009).

16. A. Abubaker and P. Van Den Berg, "Total variation as a multiplicative constraint for solving inverse problems," IEEE Trans. Image Process. 10, 1384-1392 (2001).

17. P. Guo and A. Devaney, "Comparison of reconstruction algorithms for optical diffraction tomography," J. Opt. Soc. Am. A 22, 2338-2347 (2005).

18. S. J. LaRoque, E. Y. Sidky, and X. Pan, "Accurate image reconstruction from few-view and limited-angle data in diffraction tomography," J. Opt. Soc. Am. A 25, 1772-1782 (2008).

19. L. Denis, D. Lorenz, E. Thiébaut, C. Fournier, and D. Trede, "Inline hologram reconstruction with sparsity constraints," Opt. Lett. 34, 3475-3477 (2009).

20. E. N. Leith and J. Upatnieks, "Reconstructed wavefronts and communication theory," J. Opt. Soc. Am. 52, 1123-1128 (1962).

21. U. Schnars and W. Juptner, Digital Holography, Digital Hologram Recording, Numerical Reconstruction and Related techniques (Springer-Verlag, 2005).

22. T. M. Kreis and W. P. O. Jüptner, "Suppression of the dc term in digital holography," Opt. Eng. 36, 2357-2360 (1997).

23. H. Choo, J. Woo, D. Kim, S. Shin, and Y. Yu, "DC suppression in in-line digital holographic microscopes on the basis of an intensity-averaging method using variable pixel numbers," Opt. Laser Technol. 41, 741-745 (2009).

24. J. Goodman, Introduction to Fourier Optics (McGrawHill, 1968).

25. T. Latychevskaia and H. Fink, "Solution to the twin image problem in holography," Phys. Rev. Lett. 98, 233901 (2007).

26. R. Mersereau and A. Oppenheim, "Digital reconstruction of multidimensional signals from their projections," in Proceedings of the IEEE (IEEE, 1974), pp. 1319-1338.

27. R. Mueller, M. Kaveh, and R. Inversion, "A new approach to acoustic tomography using diffraction techniques," Acoust. Imaging 8, 615-628 (1980).

28. J. Bioucas-Dias and M. Figueiredo, "A new TwIST: two-step iterative shrinkage/thresholding algorithms for image restoration," IEEE Trans. Image Process. 16, 2992-3004 (2007).

29. A. Kak and M. Slaney, Principles of Computerized Tomographic Imaging (IEEE, 1988)

30. M. A. Richards, Fundamentals of Radar Signal Processing (McGraw-Hill, 2005).

31. Z. Wu, J. Kinast, M. E. Gehm, and H. Xin, "Rapid and inexpensive fabrication of terahertz electromagnetic bandgap structures," Opt. Express 16, 16442-16451 (2008). 